\title{
Predictive value of pentraxin-3 on disease severity and mortality risk in patients with hemorrhagic fever with renal syndrome
}

Hong $\mathrm{Du}^{\dagger}$, Haifeng $\mathrm{Hu}^{\dagger}$, Pingzhong Wang ${ }^{\dagger}$, Xiaoyan Wang, Ying Zhang, Hong Jiang, Jing Li, Xuefan Bai ${ }^{*}$ and Jianqi $\operatorname{Lian}^{*}$

\begin{abstract}
Background: Hemorrhagic fever with renal syndrome (HFRS) caused by Hantaan virus is characterized by systemic immunopathological injury. Pentraxin-3 is an acute-phase reactant involved in the processes of inflammation and infection. This study aimed to investigate the levels of plasma pentraxin-3 and evaluate its predictive value on disease severity and mortality risk in patients with HFRS.

Methods: This was a prospective real-world observational study. The concentrations of plasma pentraxin-3 were measured by enzyme linked immunosorbent assay (ELISA) in 105 HFRS patients and 27 healthy controls. We analyzed the clinical relevance between pentraxin-3 and clinical subtyping, hospital stay and conventional laboratory parameters of HFRS patients. Considering the prognosis (death) as the primary endpoint, the levels of pentraxin-3 between survivors and non-survivors were compared, and its association with mortality was assessed by Kaplan-Meier survival analysis. The predictive potency of pentraxin-3 for mortality risk in HFRS patients was evaluated by receiver operating characteristic (ROC) curve analysis.

Results: The levels of pentraxin-3 during the acute phase were increased with the aggravation of the disease, and showed the highest expression in critical-type patients $(P<0.05)$. Pentraxin-3 demonstrated significant correlations with conventional laboratory parameters (WBC, PLT, AST, ALB, APTT, Fib) and the length of hospital stay. Compared with the survivors, non-survivors showed higher levels of pentraxin-3 and worse expressions of conventional laboratory parameters during the acute phase. The Kaplan-Meier survival curves showed that high levels of pentraxin-3 during the acute phase were significantly associated with the death in HFRS patients. Pentraxin-3 demonstrated significant predictive value for the mortality risk of HFRS patients, with the area under ROC curve (AUC) of 0.753 (95\%Cl: $0.593 \sim 0.914, P=0.003)$.
\end{abstract}

Conclusions: The detection of plasma pentraxin-3 might be beneficial to the evaluation of disease severity and to the prediction of mortality risk in HFRS patients.

Keywords: Hemorrhagic fever with renal syndrome, Pentraxin-3, Early prediction, Prognosis, Disease severity

\footnotetext{
* Correspondence: xfbai2011@163.com; lianjq@fmmu.edu.cn

${ }^{+}$Hong Du, Haifeng Hu and Pingzhong Wang contributed equally to this

work.

Center for Infectious Diseases, Second Affiliated Hospital of Air Force Medical

University, 569 Xinsi Rd, Baqiao District, Xi'an 710038, Shaanxi, China
}

(c) The Author(s). 2021 Open Access This article is licensed under a Creative Commons Attribution 4.0 International License, which permits use, sharing, adaptation, distribution and reproduction in any medium or format, as long as you give appropriate credit to the original author(s) and the source, provide a link to the Creative Commons licence, and indicate if changes were made. The images or other third party material in this article are included in the article's Creative Commons licence, unless indicated otherwise in a credit line to the material. If material is not included in the article's Creative Commons licence and your intended use is not permitted by statutory regulation or exceeds the permitted use, you will need to obtain permission directly from the copyright holder. To view a copy of this licence, visit http://creativecommons.org/licenses/by/4.0/ The Creative Commons Public Domain Dedication waiver (http://creativecommons.org/publicdomain/zero/1.0/) applies to the data made available in this article, unless otherwise stated in a credit line to the data. 


\section{Background}

Hemorrhagic fever with renal syndrome (HFRS) is a kind of natural focus disease caused by Hantavirus infection, and characterized by fever, hemorrhage and renal impairment [1]. Hantavirus infection could induce the destruction of vascular endothelial cells [2], the diffuse damage of systemic micro-vessels, the increase of capillary permeability and the decrease of platelets [3]. Patients with intemperate immunoreaction may further develop "capillary leakage syndrome", which could result in secondary edema, hypovolemic shock, acute kidney injury (AKI), coagulation disorder and even multiple organ dysfunction syndrome (MODS) [4]. It has been accepted that HFRS has immunopathological features of systemic inflammatory response syndrome (SIRS) [5]. The early diagnosis and disease severity assessment may help the clinicians choose the best therapeutic schedule for patients and finally improve the therapeutic effect of HFRS [6]. Given the limited predictive value of the conventional laboratory parameters, it is necessary to explore some novel biomarkers for evaluating the severity and prognosis in patients with HFRS.

As the first long-pentraxin discovered in human, pentraxin-3 is mainly produced by monocyte macrophages and myeloid dendritic cells stimulated by proinflammatory signals such as IL-1 $\beta$, TNF- $\alpha$ and Toll-like receptor activation. Additionally, a fraction of pentraxin3 is derived from the secretion of neutrophils, lymphocytes and endothelial cells [7]. Pentraxin-3 plays an important role in innate humoral immune response, inflammatory response, anti-infection, as well as tissue damage and repair [8]. Many previous studies have demonstrated that the levels of plasma pentraxin-3 were positively related to the severity of sepsis, acute pancreatitis, acute myocardial injury and other diseases, which indicated that pentraxin-3 can serve as a novel biomarker for inflammation, infection and tissue damage [9-14]. Additionally, the over-expressed pentraxin-3 of neutrophils might be associated with the overproduction of reactive oxygen species (ROS) and vascular endothelial dysfunction. Excessive pentraxin-3 might represent an emerging biomarker for the progression of vascular injury in patients with hemodialysis [15]. Previous studies have also shown that high levels of plasma pentraxin3 were associated with the development of dengue shock syndrome caused by dengue virus. Pentraxin-3 seemed to be an early prognostic indicator of disease severity in dengue hemorrhagic fever [16]. Outinen et al. [17] showed that high levels of plasma pentraxin-3 were associated with the thrombocytopenia in Puumala hantavirus (PUUV) induced nephropathia epidemica (a mild form of HFRS, mainly prevalent in the Europe). Recent study also indicated that plasma pentraxin-3 was highly correlated with PLT, Fib, APTT and other coagulation indicators, and which got a favorable predictive value for disease severity in patients with nephropathia epidemica [18]. However, the role of pentraxin-3 in HFRS caused by Hantaan virus (HTNV) has not been reported. Given the above research background, we detected the levels of plasma pentraxin-3 in patients with HFRS, and investigated its predictive value for disease severity and prognosis (death) of HFRS.

\section{Methods \\ Study population}

We prospectively recruited 105 HFRS patients who were treated in the Second Affiliated Hospital of Air Force Medical University from October 2012 to December 2014. All the enrolled patients met the following inclusion criteria: (1) Consistent with the typical clinical manifestations of HFRS and confirmed by serological examination; (2) 18-70 years old; (3) In the acute phase at the time of hospital admission. In addition, 27 healthy volunteers were recruited as controls. The patients according with any of the following criteria were excluded: (1) Age $>70$ or $<18$ years; (2) Entered the diuretic stage on admission; (3) Complicated with pregnancy, chronic kidney diseases, autoimmune diseases, bacterial infection, viral hepatitis or other infectious diseases. The study was approved by the Ethics Committee of the Second Affiliated Hospital of Air Force Medical University and the written informed consents were obtained from the participants or their guardians.

\section{Procedures and definitions}

The serological diagnosis of HFRS was confirmed by the positive results of HTNV-specific IgM/IgG capture ELIS A. According to the clinical classification criteria of HFRS [19], all the enrolled patients were divided into the following four groups: mild-type, moderate-type, severe-type and critical-type. The specific clinical typing criteria were showed in Table 1 . The classification of all patients got a confirmation from their respective attending physician. Furthermore, based upon the typical clinical characteristics of HFRS, we also divided the clinical course into the acute phase (including the febrile, hypotensive, and oliguric stages) and the convalescent phase (including the diuretic and convalescent stages) [20]. The prognosis was defined as survival or death during hospitalization.

\section{Pentraxin-3 detection and clinical data collection}

Venous blood samples of the patients were collected once each in the acute phase and convalescent phase, and were centrifuged $\left(1200 \mathrm{~g}, 10 \mathrm{~min}, 4{ }^{\circ} \mathrm{C}\right)$ within $2 \mathrm{~h}$ after drawing. Pentraxin-3 levels of supernatant plasma were measured with commercially available ELISA kits (Quantikine, XiTang Inc., Shanghai, China). Conventional laboratory 
Table 1 Grouping criteria based upon the clinical typing criteria of HFRS ${ }^{a}$

\begin{tabular}{|c|c|}
\hline Group & Clinical typing criteria of HFRS \\
\hline Mild-type & $\begin{array}{l}\text { Patients with slight symptoms and mild renal impairment (proteinuria or hematuria ranging from " }+ \text { " to " }++ \text { "), and } \\
\text { without obvious oliguria and hypotension. }\end{array}$ \\
\hline Moderate-type & $\begin{array}{l}\text { Patients with typical symptoms of effusion (bulbar conjunctiva) and petechiae (skin and mucous membranes), and } \\
\text { also meeting with obvious oliguria and kidney injury (proteinuria or hematuria more than " }+++" \text { ), some of them } \\
\text { presenting with transient hypotension. }\end{array}$ \\
\hline Severe-type & $\begin{array}{l}\text { Patients presenting with the following symptoms: } \\
\text { (1) severe effusion (bulbar conjunctiva and either pleura orperitoneum) and haemorrhage (skin and mucous } \\
\text { membranes); (2) significant hypotension and uremia;(3) AKI with oliguria (urine output } 100 \sim 500 \mathrm{~mL} / \text { day) } \leq 5 \text { days } \\
\text { or anuria (urine output }<100 \mathrm{~mL} / \text { day) } \leq 2 \text { days. }\end{array}$ \\
\hline Critical-type & $\begin{array}{l}\text { On the basis of the severe-type, patients meeting with any of the following complications: refractory shock ( } \geq 2 \text { days), } \\
\text { visceral hemorrhage, heart failure, pulmonary edema, brain edema, severe secondary infection, and severe AKI with } \\
\text { either oliguria (urine output } 100 \sim 500 \mathrm{~mL} / \text { day) }>5 \text { days or anuria (urine output }<100 \mathrm{~mL} / \text { day) }>2 \text { days. }\end{array}$ \\
\hline
\end{tabular}

Abbreviation: AKI Acute kidney injury

${ }^{a}$ The clinical typing criteria of HFRS was derived from the "Prevention and Treatment Strategy of Hemorrhagic Fever with Renal Syndrome" promulgated by the National Health Commission of China

tests (blood routine test, biochemical test and blood condensation test) were performed in the clinical laboratory as ordered by attending physicians. Data of demographic, duration of hospital stay, clinical outcome (discharge or death) and conventional laboratory parameters (blood sampling time was the same as that of pentraxin-3) were extracted from the electronic medical records.

\section{Statistical analysis}

The study was initially designed to compare the difference of plasma pentraxin-3 levels between HFRS patients and healthy controls. Our primary hypothesis was that the levels of plasma pentraxin-3 in HFRS patients would significantly higher than in healthy controls. Based upon the results of our pre-experiment, the assumed mean concentration of plasma pentraxin-3 in HFRS patients and healthy controls were $287.3 \mathrm{ng} / \mathrm{mL}$ and $8.5 \mathrm{ng} / \mathrm{mL}$, with the standard deviation of 438.5 and 13.6, respectively. Setting a 5:1 ratio of HFRS patients and healthy controls, we calculated that the original target sample size of 132 cases ( 110 HFRS patients and 22 healthy controls) would provide $81.15 \%$ power under a two-sided type I error of $5 \%$.

All the statistical analyses were performed using SPSS 23.0 software (IBM Inc., Chicago IL, USA). Continuous variables of normal distribution were presented as mean \pm standard deviation, and were compared by Student's t-test or one-way analysis of variance. Continuous variables of non-normal distribution were presented as median (interquartile range), and were compared by Mann-Whitney U test or Kruskal-Wallis $\mathrm{H}$ test. The categorical variables were presented as numbers (percentage) and were compared by chi-square test. The levels of pentraxin-3 during the acute phase and convalescent phase were compared by Wilcoxon matched-pairs signed-ranks test. Spearman correlation analysis was used to evaluate the correlations of pentraxin-3 with conventional laboratory parameters and hospital stay.
The predictive efficacy of pentraxin-3 for the prognosis (death) of HFRS patients was evaluated by the receiver operating characteristic (ROC) curve analysis and quantified by the area under ROC curve (AUC). The comparison of AUC between pentraxin-3 and conventional laboratory parameters was performed using MannWhitney U test. Kaplan-Meier survival curves with logrank test were used to assess the association of laboratory markers with mortality and calculate the hazard ratio (HR) of death. A two-sided $P<0.05$ was considered statistically significant.

\section{Results}

\section{Demographic, clinical and laboratory parameters of the study population}

From October 2012 to December 2014, a total of 386 HFRS patients were treated in our center. Of them, excluding the patients who did not meet the inclusion criteria (92 cases) and the patients who refused to participate in the study (189 cases), 105 patients with a mean age of $41.85 \pm 15.35$ years were enrolled in this prospective observational study, including 21 (20.0\%) females and $84(80.0 \%)$ males.

According to the grouping criteria mentioned above, 17 cases were classified as mild-type, 27 cases were classified as moderate-type, 26 cases were classified as severe-type, and 35 cases were classified as critical-type. Of all the enrolled patients, 14 (13.3\%) critical-type patients were died and the rest were recovered and discharged. There was no significant difference in gender, age distribution, and the timing of blood collection during the acute phase among the subgroups $(P>0.05)$ (Table 2). The length of hospital stay increased with the aggravation of the disease, while not longest in the critical-type patients, which attributed to the fact that the death usually occurred soon after admission in the non-survivors. Excluding the non-survivors, the length 


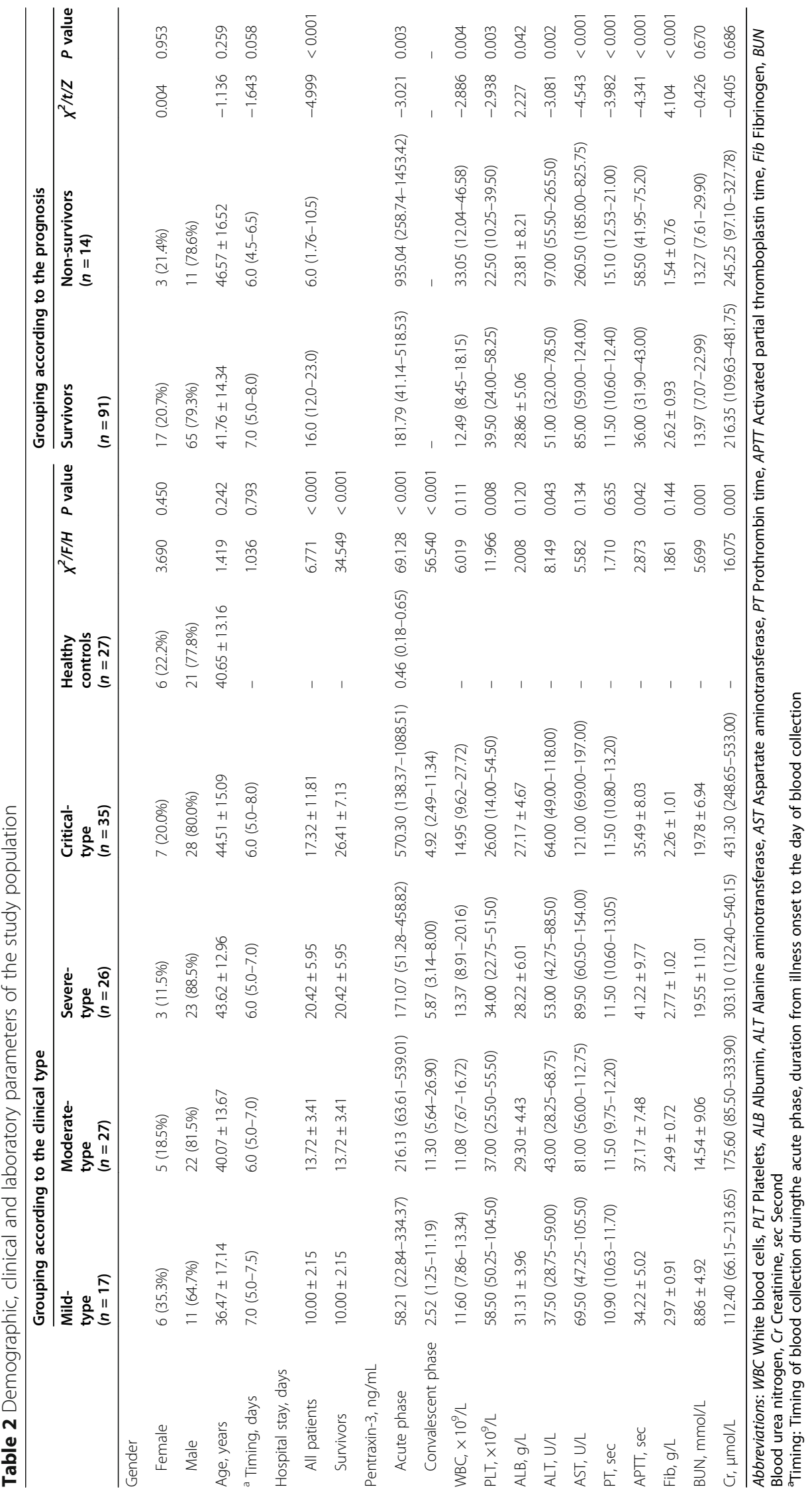


of hospital stay showed a significant correlation with disease severity (Table 2).

We comparatively analyzed the levels of pentraxin-3 among the subgroups to investigate the clinical correlation of pentraxin-3 with disease severity. Of the enrolled patients, the levels of pentraxin-3 during the acute phase were significantly higher than that of the control group and convalescent phase of the same type $(P<0.05)$. The levels of pentraxin-3 had an increasing tendency with the aggravation of the disease, and showed the highest expression in critical-type patients $(P<0.05)$. By contrast, the comparison of pentraxin-3 during the convalescent phase among the four types demonstrated no significant difference $(P<0.05)$ (Fig. 1). Compared with the survivors, non-survivors got higher levels of pentraxin-3 and worse expressions of the conventional laboratory parameters (except for the renal function markers: blood urea nitrogen and creatinine) during the acute phase. More detailed information about demographic, clinical and laboratory

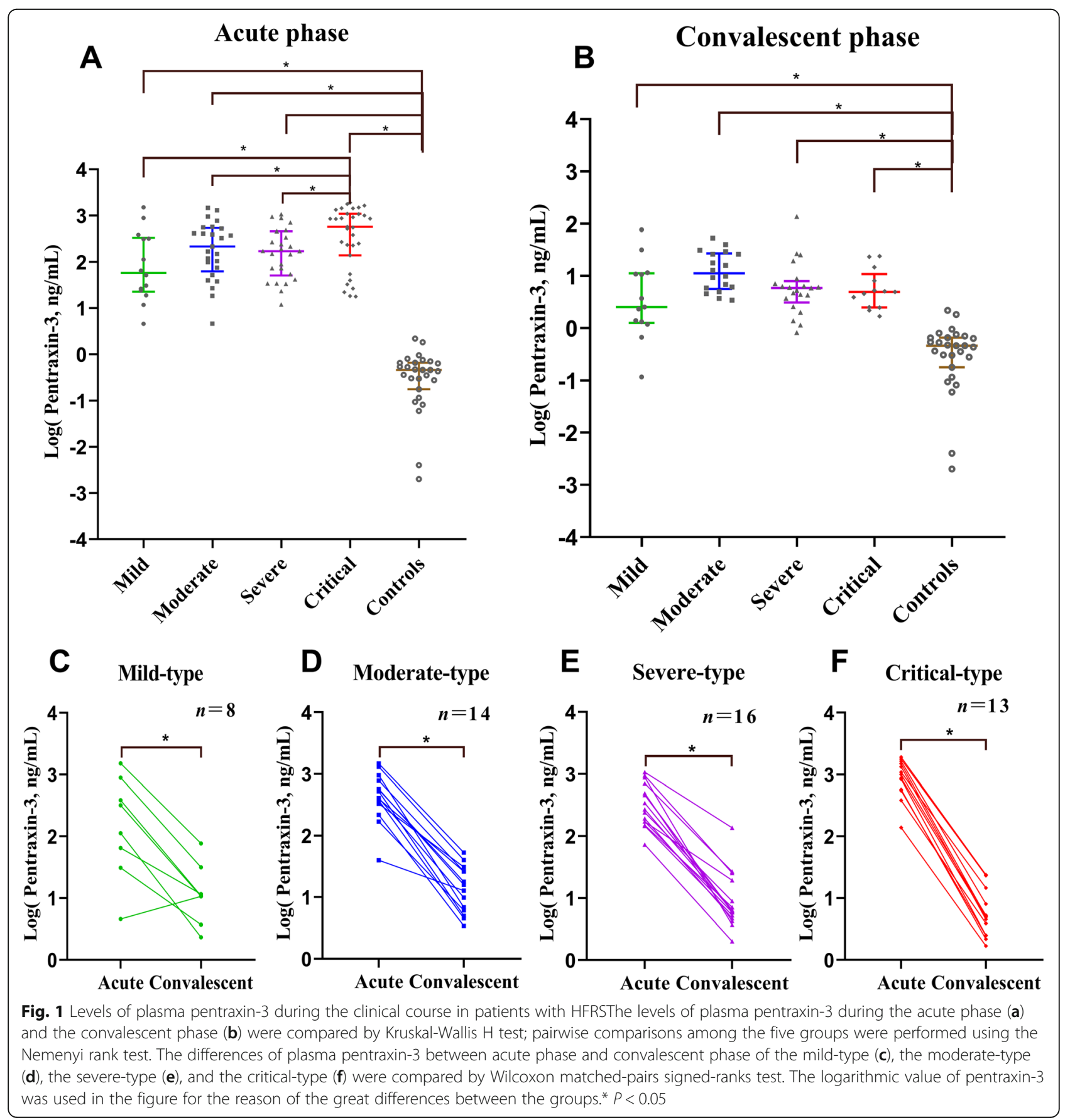




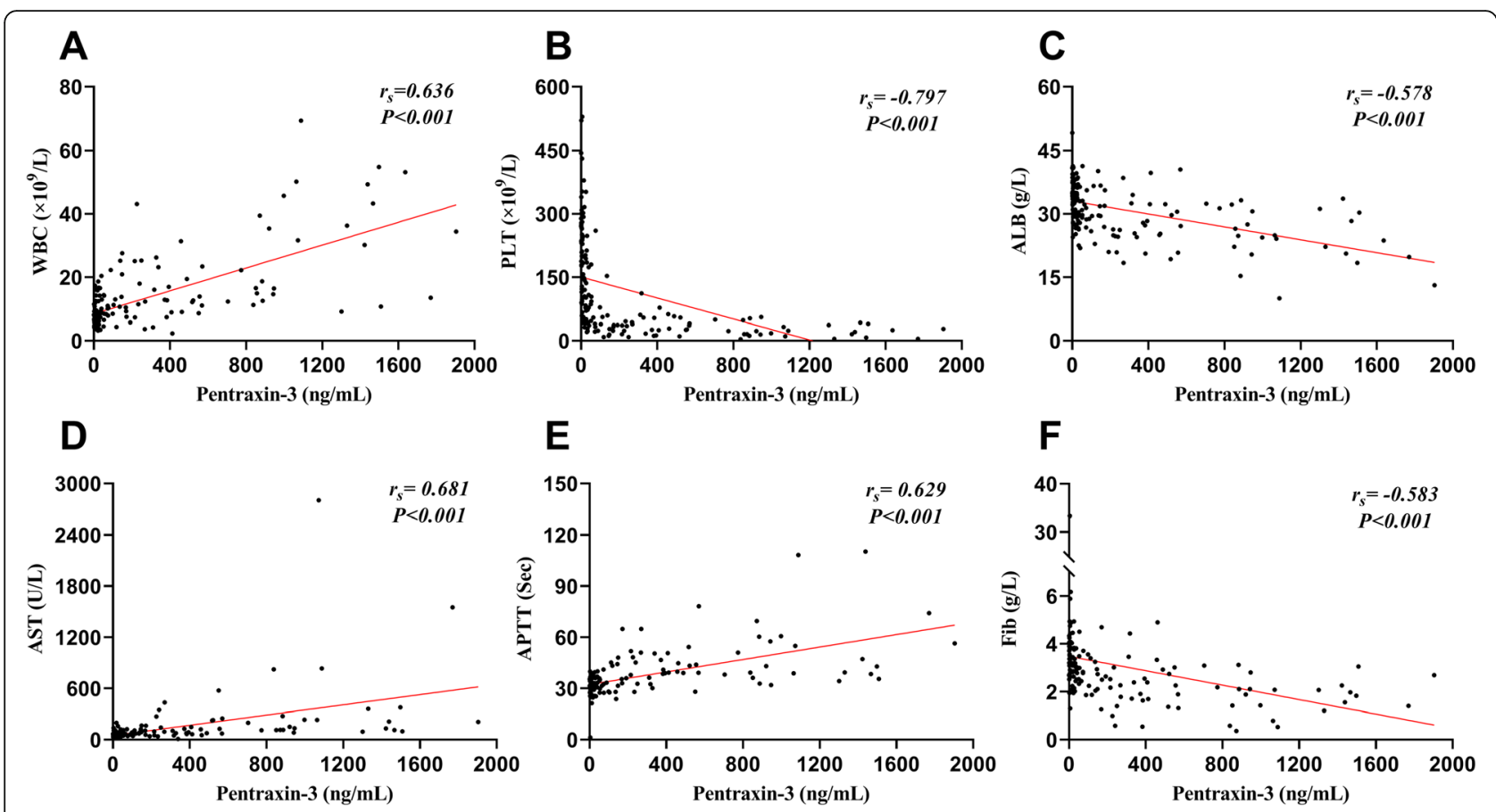

Fig. 2 The correlation between pentraxin-3 and conventional laboratory parameters. Figure shows the correlation between pentraxin-3 and WBC (a), PLT (b), ALB (c), AST (d), APTT (e), Fib (f). Spearman rank correlation analysis was used to evaluate the correlation between pentraxin-3 and conventional laboratory parameters. Abbreviations: WBC, White blood cells; PLT, Platelet; AST, Aspartate aminotransferase; ALB, Albumin; APTT, Activated partial thromboplastin time; Fib, Fibrinogen

parameters of the study population was displayed in Table 2.

The correlations of pentraxin-3 with conventional laboratory parameters and hospital stay

Spearman correlation analysis showed that pentraxin-3 was positively correlated with WBC, AST and APTT, and negatively correlated with PLT, ALB and Fib $\left(\left|r_{s}\right|>0.500\right.$, $P<0.001$ ) (Fig. 2). With hospital stay as a clinically relevant end point, pentraxin-3 and conventional laboratory parameters (WBC, PLT, AST, BUN, Cr) showed significant correlations with the length of hospital stay (Table 3).

\section{ROC curves for predictive efficacy and the hazard ratio of death}

The results of ROC curve analysis demonstrated obvious predictive value of pentraxin-3 for the prognosis (death) of HFRS patients, with the AUC of 0.753 (95\% CI: 0.593 $\sim 0.914, P=0.003$ ) (Fig. 3). Except for AST, the AUC of pentraxin-3 demonstrated no significant difference with that of conventional laboratory parameters (WBC, PLT, ALB, APTT and Fib). Taking $569.088 \mathrm{ng} / \mathrm{ml}$ as the cutoff value of pentraxin-3, the sensitivity and specificity of pentraxin-3 for predicting the prognosis (death) was 71.4 and $80.5 \%$, respectively (Table 4 ).

Furthermore, we assessed the association of laboratory parameters with mortality and calculated the hazard ratio (HR) of death using the Kaplan-Meier survival curves and log-rank test (taking death/survival as the terminal event, lengths of hospital stay as the survival time, and grouping based upon the cut-off values of ROC curves). The results showed that high levels of pentraxin-3 (>569.088 ng/mL) during the acute phase were significantly associated with the death in HFRS patients, with a HR of $7.77(2.29-26.35)$ in comparison with the low pentraxin-3 $(<569.088 \mathrm{ng} /$ mL) (Fig. 4).

\section{Discussion}

Although pentraxin-3 has been well studied in other diseases such as sepsis, acute pancreatitis and dengue hemorrhagic fever $[9,10,12,14,16]$, its expression and role has not been reported in HTNV-induced HFRS. In this study, we detected the levels of plasma pentraxin-3 in HFRS patients and healthy controls. Our results demonstrated that the expression of plasma pentraxin-3 in HFRS patients was significantly higher than that in healthy controls. Pentraxin-3 levels had an increasing tendency with the aggravation of the disease, and showed the highest expression in the critical-type patients (Table 2 and Fig. 1). The above findings indicate that pentraxin-3 can serve as an early predictor for the disease severity of HFRS, which chimes in with the results of pentraxin-3 in 
Table $3{ }^{\text {a }}$ Correlations of the laboratory parameters with pentraxin-3 and hospital stay

\begin{tabular}{|c|c|c|c|c|}
\hline \multirow{2}{*}{$\begin{array}{l}\text { Laboratory } \\
\text { parameters }\end{array}$} & \multicolumn{2}{|c|}{ Pentraxin-3, ng/mL } & \multicolumn{2}{|c|}{${ }^{\mathrm{b}}$ Hospital stay, days } \\
\hline & $r_{s}$ & $P$ value & $r_{s}$ & $P$ value \\
\hline Pentraxin-3, ng/mL & - & - & 0.232 & 0.039 \\
\hline $\mathrm{WBC}, \times 10^{9} / \mathrm{L}$ & 0.636 & $<0.001$ & 0.259 & 0.019 \\
\hline $\mathrm{PLT}, \times 10^{9} / \mathrm{L}$ & -0.797 & $<0.001$ & -0.323 & 0.003 \\
\hline$H G B, g / L$ & 0.385 & $<0.001$ & 0.057 & 0.614 \\
\hline $\mathrm{HCT}, \%$ & 0.265 & 0.001 & -0.031 & 0.784 \\
\hline$A L B, g / L$ & -0.578 & $<0.001$ & -0.180 & 0.107 \\
\hline$A L T, U / L$ & 0.303 & $<0.001$ & 0.172 & 0.135 \\
\hline AST, U/L & 0.681 & $<0.001$ & 0.249 & 0.029 \\
\hline PT, sec & 0.155 & 0.065 & 0.208 & 0.066 \\
\hline PTA, \% & -0.134 & 0.112 & -0.218 & 0.053 \\
\hline APTT, sec & 0.629 & $<0.001$ & 0.201 & 0.076 \\
\hline Fib, g/L & -0.583 & $<0.001$ & -0.156 & 0.169 \\
\hline BUN, mmol/L & 0.203 & 0.010 & 0.459 & $<0.001$ \\
\hline $\mathrm{Cr}, \mu \mathrm{mol} / \mathrm{L}$ & -0.040 & 0.618 & 0.454 & $<0.001$ \\
\hline UA, $\mu \mathrm{mol} / \mathrm{L}$ & -0.214 & 0.006 & 0.016 & 0.889 \\
\hline
\end{tabular}

Abbreviations: $r_{s}$ Coefficient of rank correlation, WBC White blood cells, PLT Platelets, HGB Hemoglobin, HCT Hematocrit, ALB Albumin, ALT Alanine aminotransferase, AST Aspartate aminotransferase, PT Prothrombin time, PTA Prothrombin activity, APTT Activated partial thromboplastin time, Fib Fibrinogen, BUN Blood urea nitrogen, $\mathrm{Cr}$ Creatinine, UA Uric acid, sec Second ${ }^{a}$ The correlations were calculated by Spearman rank correlation analysis ${ }^{\mathrm{b}}$ The data of hospital stay was derived from the survivors sepsis, acute pancreatitis, nephropathia epidemica and other diseases $[9,10,12,14,16,17]$.

Previous studies have indicated that the levels of pentraxin-3 showed significant correlations with coagulation and inflammation parameters in patients with sepsis, nephropathia epidemica, and other infectious diseases [14, 18]. Coincidentally, our study showed that pentraxin-3 was significant correlated with conventional laboratory parameters (WBC, PLT, AST, ALB, APTT, Fib) and the length of hospital stay in HFRS patients (Table 3 and Fig. 2). All the above findings further underline the role of pentraxin-3 on severity assessing in many diseases like HFRS. In addition, our study delivers an explicit result that non-survivors had higher levels of pentraxin-3 and worse expressions of the conventional laboratory parameters during the acute phase (Table 3 ). However, this was not the case for the markers of kidney function (blood urea nitrogen and creatinine) for the reason that most non-survivors died of refractory shock, rock-ribbed visceral edema and hemorrhage in the febrile and hypotensive stage. It is widely acceptable that the deterioration of kidney function markers is mainly manifested in the relatively stable oliguric stage. Therefore, the markers of kidney function exhibit hysteresis on reflecting the severity of HTNV-induced HFRS [21].

Admittedly, there is a considerable diversity and heterogeneity in the clinical course and prognosis of patients with HFRS. A marker reliably predicting the severity and prognosis of HFRS would be of clinical relevance, since it could help clinicians in individualized

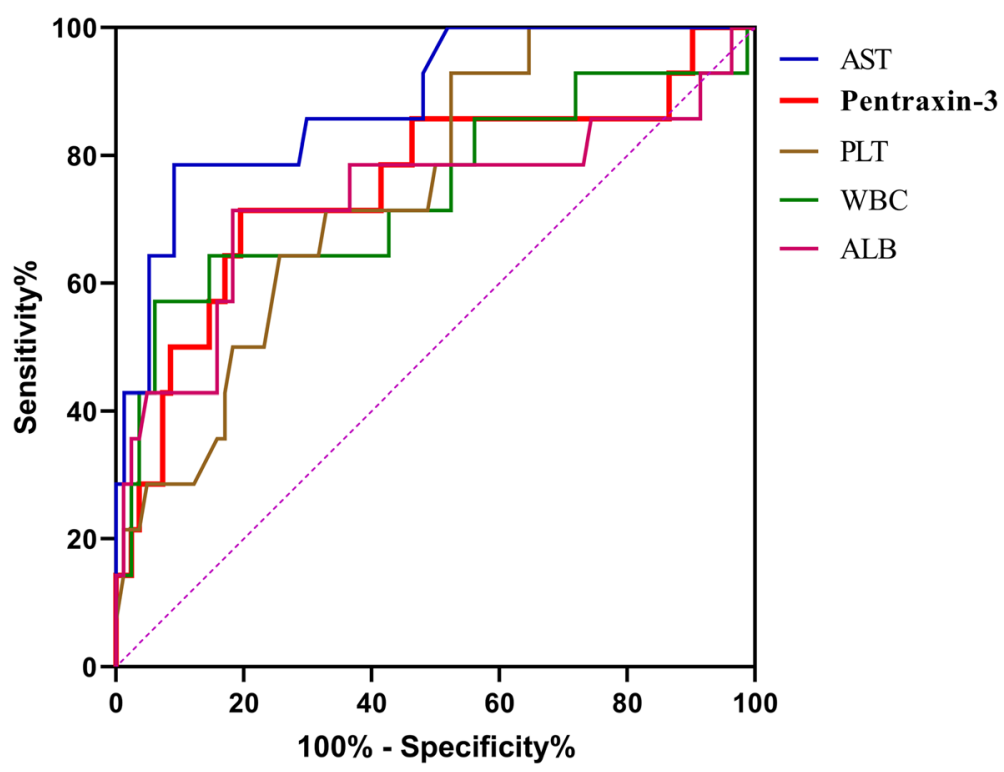

Fig. 3 ROC curves for evaluating the predictive efficacy of pentraxin-3 and conventional laboratory parameters. Figure shows the predictive efficacy of pentraxin-3, AST, PLT, WBC, and ALB for the prognosis (death) in patients with HFRS. Pentraxin-3 showed significant predictive value, with the AUC of $0.753(P=0.003)$. Abbreviations: ROC curve, Receiver operating characteristic curve; AUC, Area under the ROC curve; WBC, White blood cells; PLT, Platelet; AST, Aspartate aminotransferase; ALB, Albumin 
Table 4 Predictive efficacy of pentraxin-3 and conventional laboratory parameters

\begin{tabular}{|c|c|c|c|c|c|c|c|}
\hline & \multirow[t]{2}{*}{ AUC $(95 \% C l)$} & \multirow{2}{*}{$\begin{array}{l}P \\
\text { value }\end{array}$} & \multirow{2}{*}{$\begin{array}{l}\text { Cut-off } \\
\text { value }\end{array}$} & \multirow[t]{2}{*}{ Sensitivity } & \multirow[t]{2}{*}{ Specifity } & \multicolumn{2}{|c|}{ Comparison of AUC (pentraxin-3) } \\
\hline & & & & & & $\bar{Z}$ & $P$ value \\
\hline Pentraxin-3, ng/mL & $0.753(0.593-0.914)$ & 0.003 & 569.088 & $71.4 \%$ & $80.5 \%$ & - & - \\
\hline $\mathrm{WBC}, \times 10^{9} / \mathrm{L}$ & $0.742(0.573-0.911)$ & 0.004 & 31.525 & $57.1 \%$ & $93.9 \%$ & 0.188 & 0.851 \\
\hline $\mathrm{PLT}, \times 10^{9} / \mathrm{L}$ & $0.747(0.621-0.872)$ & 0.003 & 41.5 & $47.6 \%$ & $92.9 \%$ & 0.110 & 0.912 \\
\hline$A L B, g / L$ & $0.732(0.553-0.911)$ & 0.006 & 24.85 & $81.7 \%$ & $71.4 \%$ & 0.344 & 0.731 \\
\hline AST, U/L & $0.883(0.787-0.979)$ & $<0.001$ & 203 & $78.6 \%$ & $90.9 \%$ & 2.163 & 0.031 \\
\hline APTT, sec & $0.865(0.747-0.984)$ & $<0.001$ & 54.55 & $64.3 \%$ & $96.2 \%$ & 1.670 & 0.095 \\
\hline Fib, g/L & $0.824(0.710-0.937)$ & $<0.001$ & 1.8425 & $86.1 \%$ & $71.4 \%$ & 0.785 & 0.433 \\
\hline $\mathrm{Cr}, \mu \mathrm{mol} / \mathrm{L}$ & $0.534(0.391-0.677)$ & 0.686 & - & - & - & - & - \\
\hline BUN, mmol/L & $0.536(0.359-0.712)$ & 0.670 & - & - & - & - & - \\
\hline
\end{tabular}

Abbreviations: AUC Area under the ROC curve, Cl Confidence interval, WBC White blood cells, PLT Platelets, ALB Albumin, AST Aspartate aminotransferase, APTT Activated partial thromboplastin time, Fib Fibrinogen, $\mathrm{Cr}$ Creatinine, BUN Blood urea nitrogen, sec Second

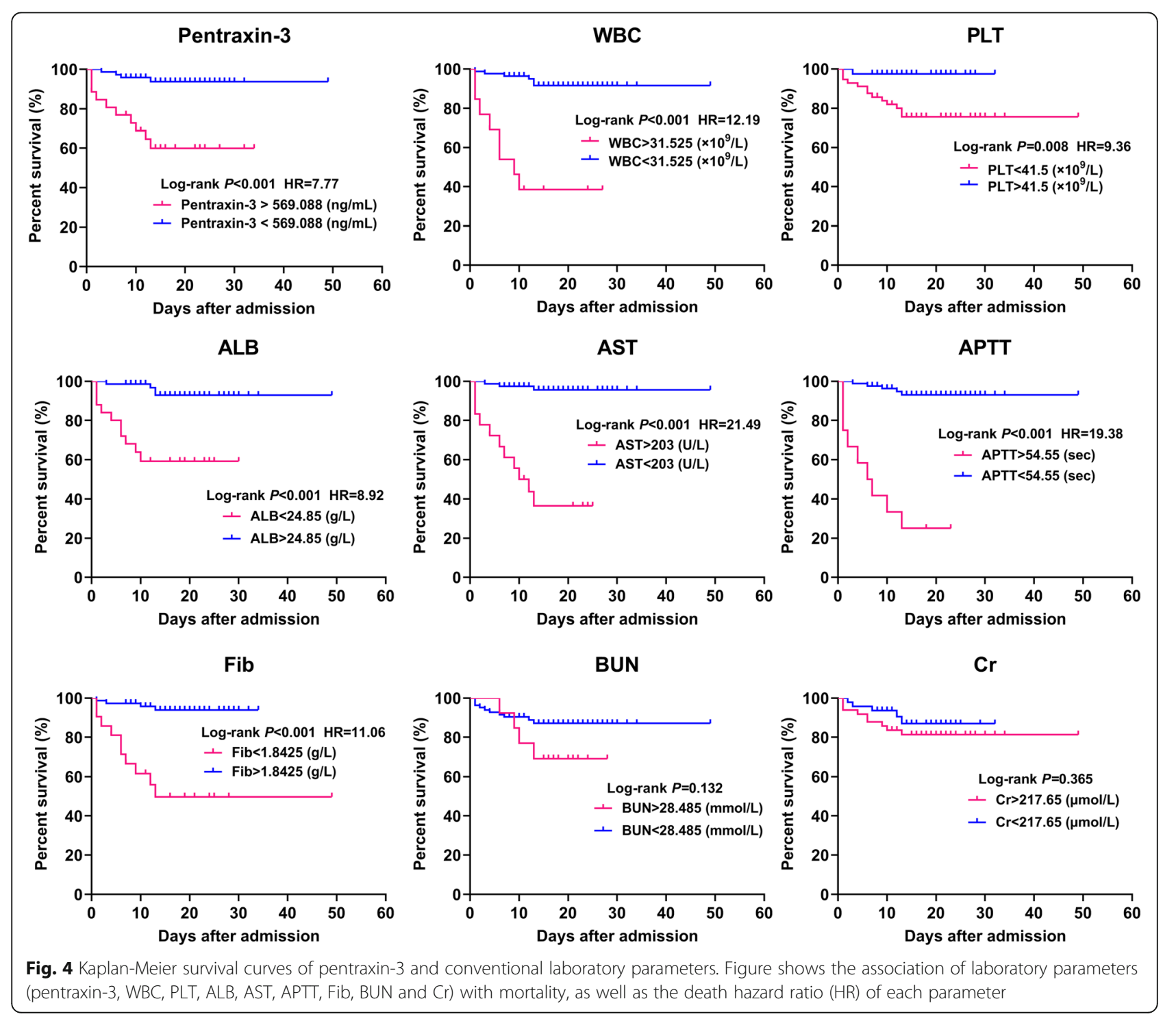


therapy allocation based on the disease severity. In recent years, some studies have identified several early predictors related to the severity and prognosis of HFRS, such as growth arrest-specific 6 protein, urinary neutrophil gelatinase-associated lipocalin and Glycoprotein YKL-40 [20-22]. In this study, we observed that high levels of pentraxin-3 ( $>569.088 \mathrm{ng} / \mathrm{mL})$ during the acute phase were significantly associated with the death in HFRS patients (Fig. 4). Pentraxin-3 also demonstrated a significant predictive value for the prognosis (death) of HFRS patients, and which was comparable with the predictive value of conventional laboratory parameters such as PLT (Table 4 and Fig. 3). To sum up, pentraxin-3 could serve as a novel and efficient biomarker for predicting the disease severity and mortality risk in patients with HFRS.

As one of the most important immune cells participating in innate immunity, neutrophils play a crucial part in resisting bacterial infection. Nevertheless, the neutrophils are also elevated in most HFRS patients during the acute phase, and also positively correlated with the disease severity of HFRS [19, 23]. Therefore, hyperactive neutrophils may also participate in the immunopathological injury of HFRS by releasing neutrophilic granules. Pentraxin-3 synthesized by neutrophils is mainly stored in neutrophilic granules, which can interact with a variety of bacteria, fungi and viruses after release and then propel the phagocytosis and clearance of pathogenic microorganisms [24]. As an important component of innate humoral immunity, pentraxin-3 has the ability to bind complement component $\mathrm{C} 1 \mathrm{q}$ and then activate the classical pathway of complement $[25,26]$. Recently, many studies have showed that pentraxin-3 could modulate inflammatory cells, interact with P-selectin, reduce the nitric oxide $(\mathrm{NO})$ synthesis of endothelial cells, inhibit endothelial cells proliferation and alter their functions, and finally promote vascular inflammatory response and endothelial dysfunction [27, 28]. Therefore, the release of pentraxin-3 by neutrophil degranulation may be an important link in the immunopathological injury of HFRS, and the level of plasma pentraxin-3 may also indirectly reflect the severity of vascular endothelial injury in patients with HFRS. However, the underlying pathomechanism of endothelial injury and vascular leakage in HTNV-induced HFRS is still not fully understood. Consequently, further studies are essential to clarify the role of pentraxin-3 on the pathological injury of HFRS and its related mechanism.

As an observational study, although we got a meaningful conclusion that pentraxin-3 could serve as an efficient biomarker for predicting the disease severity and mortality risk of HFRS, there were still some limitations. First, this study was conducted in a single center for infectious diseases. The results might be limited by the relatively small sample size because of the gradually declining incidence of HFRS in Xi'an city. For the reason that several mild-type patients tided over the acute phase instantly after admission and only convalescent samples available, and the decedent died before entering the convalescence phase, only 96 venous blood specimens during the acute phase and 65 during the convalescent phase were collected from the enrolled patients. All these adverse factors may affect the accuracy of the results. Second, the definition of blood sample collection time was too broad in this study. Given the individual differences of patients' condition and the clinical process on admission, we could only collect venous blood specimens according to the acute phase and convalescent phase defined in the study. Although there was no significant statistical difference on sample collection time of the acute phase, the levels of plasma pentraxin-3 might still be influenced by the different time-points, and also by the variability of pathological injury during the acute phase of HFRS. Third, it is essential to conduct a prospective, large sample, multicenter cohort study to further confirm the predictive efficacy and clinical application value of plasma pentraxin-3 for disease severity and prognosis (death) in HFRS patients. Last but not least, the research limitations caused by the experimental measurement errors and the outdated clinical typing criteria should not be overlooked.

\section{Conclusions}

Pentraxin-3 could serve as a novel and efficient biomarker for predicting the disease severity and mortality risk of patients with HFRS. The detection of plasma pentraxin-3 might help clinicians quickly identify the severe patients at an early stage and timely take optimal therapeutic schedule for them, so as to improve the therapeutic effect and the prognosis of HFRS.

\section{Abbreviations}

HFRS: Hemorrhagic fever with renal syndrome; AKI: Acute kidney injury; MODS: Multiple organ dysfunction syndrome; SIRS: Systemic inflammatory response syndrome; ROS: Reactive oxygen species; PUUV: Puumala hantavirus; HTNV: Hantaan virus; ELISA: Enzyme linked immunosorbent assay; ROC curve: Receiver operating characteristic curve; AUC: Area under the ROC curve; WBC: White blood cells; PLT: Platelet; HGB: Hemoglobin;

HCT: Hematocrit; ALB: Albumin; ALT: Alanine aminotransferase; AST: Aspartate aminotransferase; PT: Prothrombin time; PTA: Prothrombin activity;

APTT: Activated partial thromboplastin time; Fib: Fibrinogen; BUN: Blood urea nitrogen; Cr: Creatinine; UA: Uric acid

\section{Acknowledgements}

We acknowledge all the enrolled patients and healthy volunteers, and also the health-care workers involved in the diagnosis and treatment of patients with HFRS.

\section{Authors' contributions}

JQL and XFB conceived and designed the study and had full access to all of the data in the study, and they take responsibility for the integrity of the data and the accuracy of the data analysis. HD, XYW and JL collected the venous blood samples and detected the levels of plasma pentraxin-3 using the ELISA test. HFH and XYW collected the clinical data of all enrolled patients. HFH and HD conducted the statistical analysis and manuscript drafting. PZW, YZ, HJ, XFB and JQL reviewed the manuscript and made critical 
revision. All authors have made substantial contributions to this study, and all of them read and approved the final manuscript.

\section{Funding}

This study was funded by the National Science and Technology Major Project (grant number 2017ZX10204401-002-005); the Key Clinical Research Project of Technology Innovation and Development Foundation in the Second Affiliated Hospital of Air Force Medical University (grant number 2019LCYJ002); and the General Clinical Research Project of Technology Innovation and Development Foundation in the Second Affiliated Hospital of Air Force Medical University (grant number 2019LCYJ011). The funders had no role in the design and conduct of the study.

\section{Availability of data and materials}

The datasets used and/or analyzed during the current study are available from the corresponding author on reasonable request.

\section{Declarations}

\section{Ethics approval and consent to participate}

This study was approved by the ethics committee of the Second Affiliated Hospital of Air Force Medical University and was performed in accordance with the Helsinki Declaration. Before inclusion, the patients and healthy volunteers were informed about the objectives of this study, and they agreed and signed the informed consent form. For the reason that a few critical HFRS patients had developed consciousness disorders on admission, or received mechanical ventilation and treated by sedative like midazolam, so their legal guardians signed the informed consent form on their behalf.

\section{Consent for publication}

Not applicable.

\section{Competing interests}

The authors declare that they have no competing interests.

Received: 21 May 2020 Accepted: 5 May 2021

Published online: 17 May 2021

\section{References}

1. Avšič-Županc T, Saksida A, Korva M. Hantavirus infections. Clin Microbiol Infect. 2019;21S:e6-e16. https://doi.org/10.1111/1469-0691.12291.

2. Mackow ER, Gavrilovskaya IN. Hantavirus regulation of endothelial cell functions. Thromb Haemost. 2009;102(6):1030-41. https://doi.org/10.1160/ TH09-09-0640.

3. Mittler E, Dieterle ME, Kleinfelter LM, Slough MM, Chandran K, Jangra RK. Hantavirus entry: perspectives and recent advances. Adv Virus Res. 2019;104: 185-224. https://doi.org/10.1016/bs.aivir.2019.07.002.

4. Vaheri A, Strandin T, Hepojoki J, Sironen T, Henttonen H, Mäkelä S, et al. Uncovering the mysteries of hantavirus infections. Nat Rev Microbiol. 2013; 11(8):539-50. https://doi.org/10.1038/nrmicro3066.

5. Garanina E, Martynova E, Davidyuk Y, Kabwe E, Ivanov K, Titova A, et al. Cytokine storm combined with Humoral immune response defect in fatal hemorrhagic fever with renal syndrome case, Tatarstan, Russia. Viruses. 2019; 11(7). https://doi.org/10.3390/v11070601.

6. Jiang H, Du H, Wang LM, Wang PZ, Bai XF. Hemorrhagic fever with renal syndrome: pathogenesis and clinical picture. Front Cell Infect Microbiol. 2016;6:1.

7. Bottazzi B, Garlanda C, Cotena A, Moalli F, Jaillon S, Deban L, et al. The long pentraxin PTX3 as a prototypic humoral pattern recognition receptor: interplay with cellular innate immunity. Immunol Rev. 2009;227(1):9-18. https://doi.org/10.1111/j.1600-065X.2008.00719.X

8. Garlanda C, Jaillon S, Doni A, Bottazzi B, Mantovani A. PTX3, a humoral pattern recognition molecule at the interface between microbe and matrix recognition. Curr Opin Immunol. 2016;38:39-44. https://doi.org/10.1016/j. coi.2015.11.002.

9. Albert Vega C, Mommert M, Boccard M, Rimmelé T, Venet F, Pachot A, et al. Source of circulating Pentraxin 3 in septic shock patients. Front Immunol. 2018;9:3048.

10. Song J, Park DW, Moon S, Cho HJ, Park JH, Seok H, et al. Diagnostic and prognostic value of interleukin-6, pentraxin 3, and procalcitonin levels among sepsis and septic shock patients: a prospective controlled study according to the Sepsis-3 definitions. BMC Infect Dis. 2019;19(1):968. https:// doi.org/10.1186/s12879-019-4618-7.

11. Ninomiya I, Kanazawa M, Umemura M, Onodera O. Elevated serum pentraxin 3 levels might predict the diagnosis of branch atheromatous disease at a very early stage. Eur J Neurol. 2020;27(7):1279-84. https://doi. org/10.1111/ene.14249.

12. Lee $Y T$, Gong M, Chau A, Wong WT, Bazoukis G, Wong SH, et al. Pentraxin-3 as a marker of sepsis severity and predictor of mortality outcomes: a systematic review and meta-analysis. J Inf Secur. 2018;76:1-10.

13. Zagidullin N, Motloch LJ, Gareeva D, Hamitova A, Lakman I, Krioni I, et al. Combining novel biomarkers for risk stratification of two-year cardiovascular mortality in patients with ST-elevation myocardial infarction. J Clin Med. 2020;9(2). https://doi.org/10.3390/jcm9020550.

14. Mauri T, Bellani G, Patroniti N, Coppadoro A, Peri G, Cuccovillo I, et al. Persisting high levels of plasma pentraxin 3 over the first days after severe sepsis and septic shock onset are associated with mortality. Intensive Care Med. 2010;36(4):621-9. https://doi.org/10.1007/s00134-010-1752-5.

15. dell'Oglio MP, Simone S, Ciccone M, Corciulo R, Gesualdo M, Zito A, et al. Neutrophil-dependent pentraxin-3 and reactive oxygen species production modulate endothelial dysfunction in haemodialysis patients. Nephrol Dial Transplant. 2017;32:1540-9.

16. Mairuhu AT, Peri G, Setiati TE, Hack CE, Koraka P, Soemantri A, et al. Elevated plasma levels of the long pentraxin, pentraxin 3 , in severe dengue virus infections. J Med Virol. 2005;76(4):547-52. https://doi.org/10.1002/jmv.20397.

17. Outinen TK, Mäkelä S, Huhtala H, Hurme M, Meri S, Pörsti l, et al. High pentraxin-3 plasma levels associate with thrombocytopenia in acute Puumala hantavirus-induced nephropathia epidemica. Eur J Clin Microbiol Infect Dis. 2012;31(6):957-63. https://doi.org/10.1007/s10096-011-1392-x.

18. Laine OK, Koskela SM, Outinen TK, Joutsi-Korhonen L, Huhtala H, Vaheri A, et al. Plasma pentraxin-3 and coagulation and fibrinolysis variables during acute Puumala hantavirus infection and associated thrombocytopenia. Blood Coagul Fibrinolysis. 2014;25(6):612-7. https://doi.org/10.1097/MBC. 0000000000000117

19. Du H, Li J, Yu HT, Jiang W, Zhang Y, Wang JN, et al. Early indicators of severity and construction of a risk model for prognosis based upon laboratory parameters in patients with hemorrhagic fever with renal syndrome. Clin Chem Lab Med. 2014;52(11):1667-75. https://doi.org/10.151 5/cclm-2014-0016.

20. Zhang C, Tang K, Zhang Y, Ma Y, Zhuang R, Zheng X, et al. Elevated plasma growth arrest-specific 6 protein levels are associated with the severity of disease during Hantaan virus infection in humans. Viral Immunol. 2017;30(5): 330-5. https://doi.org/10.1089/vim.2016.0137.

21. Bunz H, Weyrich P, Peter A, Baumann D, Tschritter O, Guthoff M, et al. Urinary Neutrophil Gelatinase-Associated Lipocalin (NGAL) and proteinuria predict severity of acute kidney injury in Puumala virus infection. BMC Infect Dis. 2015;15(1):464. https://doi.org/10.1186/s12879-015-1180-9.

22. Outinen TK, Mantula $P$, Jaatinen $P$, Hämäläinen $M$, Moilanen $E$, Vaheri $A$, et al. Glycoprotein YKL-40 is elevated and predicts disease severity in Puumala hantavirus infection. Viruses. 2019;11(9):767. https://doi.org/10.3390/v11090767.

23. Strandin T, Mäkelä S, Mustonen J, Vaheri A. Neutrophil activation in acute hemorrhagic fever with renal syndrome is mediated by hantavirus-infected microvascular endothelial cells. Front Immunol. 2018;9:2098. https://doi. org/10.3389/fimmu.2018.02098.

24. Razvina O, Jiang S, Matsubara K, Ohashi R, Hasegawa G, Aoyama T, et al. Differential expression of pentraxin 3 in neutrophils. Exp Mol Pathol. 2015; 98(1):33-40. https://doi.org/10.1016/j.yexmp.2014.11.009.

25. Daigo K, Inforzato A, Barajon I, Garlanda C, Bottazzi B, Meri S, et al. Pentraxins in the activation and regulation of innate immunity. Immunol Rev. 2016:274(1):202-17. https://doi.org/10.1111/imr.12476.

26. Haapasalo K, Meri S. Regulation of the complement system by pentraxins. Front Immunol. 2019;10:1750. https://doi.org/10.3389/fimmu.2019.01750.

27. Carrizzo A, Lenzi P, Procaccini C, Damato A, Biagioni F, Ambrosio M, et al. Pentraxin 3 induces vascular endothelial dysfunction through a P-selectin/ matrix Metalloproteinase-1 pathway. Circulation. 2015;131(17):1495-505. https://doi.org/10.1161/CIRCULATIONAHA.114.014822.

28. Zlibut A, Bocsan IC, Agoston-Coldea L. Pentraxin-3 and endothelial dysfunction. Adv Clin Chem. 2019;91:163-79. https://doi.org/10.1016/bs.acc.2019.03.005.

\section{Publisher's Note}

Springer Nature remains neutral with regard to jurisdictional claims in published maps and institutional affiliations. 